\title{
Pontos de carbono luminescentes à base de ovalbumina aplicados em marcação biológica
}

\author{
Luminescent carbon dots based on ovalbumin \\ applied as biological markers
}

\author{
Sarah Lima ${ }^{1}$, Luis Fernando T. Domingos ${ }^{1}$, Roberto Vaz ${ }^{1}$, \\ Paulo Henrique de Almeida Campos Junior ${ }^{2}$, Marco Antônio Schiavon ${ }^{1}$
}

\begin{abstract}
${ }^{1}$ Grupo de Pesquisa em Química de Materiais, Departamento de Ciências Naturais da Universidade Federal de São João del-Rei, Praça Dom Helvécio, 74, Campus Dom Bosco, CEP: 36301-160, São João del-Rei, MG, Brasil.

${ }^{2}$ Núcleo de Criação de Animais de Laboratório, Departamento de Ciências Naturais da Universidade Federal de São João del-Rei, Praça Dom Helvécio, 74, Campus Dom Bosco, CEP: 36301-160, São João del-Rei, MG, Brasil. e-mail: schiavon@ufsj.edu.br, paulohenrique@ufsj.edu.br
\end{abstract}

\section{RESUMO}

Entre os nanomateriais de carbono, os pontos de carbono (PCs) estão entre os mais atraentes devido as suas interessantes propriedades fotoluminescentes. Os PCs apresentam processo de síntese fácil, possibilidade de funcionalização da superfície, baixa toxicidade, alta solubilidade em água e biocompatibilidade. Devido a isso, suas aplicações na área biomédica e de bioimagem tem se destacado na literatura. Neste trabalho, PCs foram sintetizados por dois métodos diferentes a partir da ovalbumina, o qual é um precursor não tóxico e composto predominantemente por aminoácidos de cadeia curta. Os PCs foram caracterizados por espectroscopias de absorção (UV-Vis), de emissão (fluorescência) e infravermelho (IVTF), além de análise termogravimétrica (ATG). Os PCs foram utilizados como sondas fluorescentes para uma imagem colorida (azul e verde) de células HEK293 e não induziram a morte celular, o que indica que os mesmos são biocompatíveis e não tóxicos para este tipo de células. Dessa forma, os resultados aqui apresentados demonstram que os PCs podem ser considerados potenciais substitutos para corantes comerciais em marcação biológica.

Palavras-chave: nanopartículas de carbono, fotoluminescência, biocompatibilidade, HEK293.

\begin{abstract}
Among the carbon nanomaterials, carbon dots (CDs) are among the most attractive ones due to their interesting photoluminescent properties. PCs feature easy synthesis process, possibility of surface functionalization, low toxicity, high water solubility and biocompatibility. Because of this, their applications in the biomedical and bioimaging areas has been highlighted in literature. In this work, CDs were synthesized by two different methods using ovalbumin as precursor, which is a nontoxic reactant composed predominantly of short chain amino acids. The CDs were characterized by absorption (UV-Vis), emission (fluorescence), infrared (IRFT) spectroscopies, and thermogravimetric analysis (TGA). The CDs were used as fluorescent probes for multicolor (blue and green) imaging of HEK293 cells and did not induce to cell death, which indicates that the CDs are biocompatible and nontoxic to this type of cells. Therefore, the results presented here demonstrated that CDs can be considered as a potential substitute for commercial dyes in biological images.
\end{abstract}

Keywords: carbon nanoparticles, fluorescence, biocompatibility, HEK293. 


\section{INTRODUÇÃO}

O carbono é um material geralmente encontrado na coloração preta e, antes da expansão da nanotecnologia, acreditava-se que ele era insolúvel em água e não apresentava luminescência. Além de ser um dos elementos mais abundantes presentes na crosta terrestre, o carbono chama a atenção por sua versatilidade química, que lhe proporciona organização e propriedades que são únicas. Os nanomateriais de carbono, marcam os avanços na nanotecnologia e representam grande parte desse crescimento tecnológico. Dentre esses nanomateriais, devido as suas propriedades ópticas, físicas e químicas peculiares, os pontos de carbono (PCs) ganham a cada dia mais destaque na literatura [1-3].

Os PCs são nanomateriais fotoluminescentes de morfologia quase esférica, com diâmetros inferiores a 10 $\mathrm{nm}$, que foram descobertos acidentalmente em 2004 pela purificação de nanotubos de carbono de parede única por eletroforese [1, 4]. São nanopartículas de natureza não-cristalina, formadas por carbonos $s p^{2} / s p^{3}$ e grupos contendo oxigênio [5]. A principal razão dos PCs atraírem grande interesse para aplicações tecnológicas é devido às suas propriedades excepcionais, tais como: elevada solubilidade em água, inércia química, possibilidade de funcionalização, resistência à fotodegradação, baixa toxicidade, biocompatibilidade e intensa fliorescência, geralmente na região do azul do espectro eletromagnético. Essas propriedades os tornam excelentes para aplicações em diversas áreas: como o uso em optoeletrônica, dispositivos fotovoltaicos, fotocatálise, uso na biotecnologia, sensores, dentre outros [6,7].

Os PCs que contém apenas oxigênio, carbono e hidrogênio em sua composição geralmente, apresentam baixa luminescência o que pode limitar suas aplicações. Os grupos de superfície influenciam muito nas propriedades fluorescentes, e esses parâmetros podem ser otimizados a partir de estratégias de funcionalização: a dopagem com heteroátomos e a passivação de superfície com grupos funcionais específicos. A dopagem com heteroátomos pode ser feita utilizando precursores que apresentem tais elementos em sua composição. A adição de heteroátomos afeta as propriedades fluorescentes pois, mudam a distribuição dos elétrons e, consequentemente, variam o bandgap do material. Os elementos mais utilizados para a dopagem são o nitrogênio (a dopagem mais relatada na literatura), seguida por enxofre, boro e fósforo [7]. A passivação da superfície é um processo mais oneroso e necessita de duas ou mais etapas para a realização. Normalmente, o ligante utilizado para a modificação se liga aos grupos de superfície dos PCs através de interações eletrostáticas e coordenação. Os ligantes funcionais mais relatados na literatura são: polímeros, DNA, íons e proteínas [5, 7, 8].

Frente às limitações dos tradicionais pontos quânticos semicondutores, como a alta toxicidade associada à presença de metais tóxicos, os PCs apresentam propriedades ópticas ajustáveis e ainda apresentam biocompatibilidade. Assim, estudos utilizando PCs em aplicações biológicas, vem crescendo exponencialmente ao longo dos últimos anos. Existem estudos relatados na literatura da utilização tanto em bioimagem quanto na nanomedicina em entrega controlada de fármacos [9-11]. DENG et al., por exemplo, utilizaram pontos de carbono para desenvolver uma nanoplaca para fornecimento de óxido nítrico. Esse dispositivo tem a capacidade de direcionar o alvo, rastrear a fluorescência e fornecer a entrega do medicamento de forma controlada através da luz [12]. HU et al. sintetizaram PCs, em temperatura ambiente a partir de albumina pelo método micro-ondas, para utilização em imagens biológicas. Obtiveram boa biocompatibilidade e os resultados mostraram-se adequados na utilização de PCs em imagiologia celular [13].

Em bioimagem os PCs são utilizados em substituição a materiais consolidados na literatura como: complexos de porfirina, rodamina e pontos quânticos (por exemplo: CdTe, CdSe e ZnS). Novamente, os PCs são utilizados devido à baixa toxicidade, capacidade de rastreamento em tempo real de células vivas, emissão de luz em diferentes comprimentos de onda somente com a variação do comprimento de onda de excitação e luminescência estável em longos intervalos de tempo. Em geral os PCs se movem para dentro das células provavelmente por endocitose (processo no qual uma substância entra em uma célula por meio da membrana celular). Os PCs podem ser absorvidos por alguns peptídeos e ter o transporte facilitado para a membrana plasmática. [14-16]

As células HEK293, linha celular do rim embrionário humano, são utilizadas devido a sua alta reprodutibilidade, facilidade de cultivo e transfecção, sendo utilizadas na produção de proteínas e drogas e, por isso, são largamente utilizadas em estudos científicos [17]. Assim, este trabalho teve como objetivo sintetizar PCs por duas rotas distintas, utilizando ovalbumina como precursor, para utilizá-los como sondas fluorescentes para marcação de células HEK293.

\section{MATERIAIS E MÉTODOS}

\subsection{Materiais}

Foram utilizados para as sínteses dos PCs ovalbumina (Sigma Aldrich, 99\%) e água ultrapura, com resistividade elétrica menor que $18,2 \mathrm{M} \Omega / \mathrm{cm}$, a $25^{\circ} \mathrm{C}$. As células do rim embrionário humano 293 (HEK 293) foram obtidas, descongeladas e cultivadas de acordo com as instruções do fornecedor HEK293.2sus (ATCC® 
CRL1573.3 ${ }^{\mathrm{TM}}$, Virginia, EUA). Também foram utilizados solução salina tamponada com solvente - PBS (fosfato salino) (Ingamed, PR, Brasil), tampão Formalina (Sigma Aldrich - 10\%) e TRITON X-100 (Sigma Aldrich - 10\%). Além disso, foi utilizado o marcador nuclear DAPI (Sigma Aldrich), também de acordo com as instruções do fornecedor.

\subsection{Metodologia}

Tomando a ovalbumina como fonte de carbono, os PCs foram preparados utilizando os métodos de pirólise e síntese hidrotérmica. Na síntese via pirólise, $1,0 \mathrm{~g}$ de ovalbumina foi pesada e levada a um forno do tipo tubular a diferentes temperaturas $\left(200,300,400,500\right.$ e $\left.600^{\circ} \mathrm{C}\right)$, por $120 \mathrm{~min}$ em atmosfera inerte. A atmosfera inerte foi mantida por meio de um fluxo (150mL/min) de gás argônio (99,995\% de pureza) no forno tubular usado. Após a carbonização, realizou-se o processo de trituração para a obtenção de um pó finamente dividido que, na sequência, foi transferido para um béquer contendo $100 \mathrm{~mL}$ de água ultrapura. Logo após, com o intuito de extrair nanopartículas que ainda estavam adsorvidas no carvão, a solução foi levada a um banho de ultrassom por $30 \mathrm{~min}$. Em seguida, a mesma foi condicionada a agitação e aquecimento, a uma temperatura de $100^{\circ} \mathrm{C}$, por $60 \mathrm{~min}$. Por fim, a solução foi centrifugada a $3600 \mathrm{rpm}$ por 15 min e filtrada a vácuo, descartando-se o precipitado. No método hidrotérmico, a mesma quantidade do precursor foi pesada e adicionada a um recipiente de Teflon, juntamente com $50 \mathrm{~mL}$ de água ultrapura. O recipiente foi colocado no interior de um Reator Autoclave em uma temperatura fixada em $150^{\circ} \mathrm{C}$, variando-se o tempo de síntese em 2, 5, 10 e 20 h. Neste caso, a suspensão dos PCs foi obtida após um processo de centrifugação (3600 rpm, 15 min) e filtragem à vácuo.

Para a análise da marcação celular, as células foram fixadas utilizando tampão neutro de Formalina por 20 min. Depois disso, essas células foram divididas em dois grupos: a) permeabilizadas com 1\% de Triton X-100 em PBS (concentração de $0,10 \mathrm{molL}^{-1}$ ) pH 7,5 por 20 min e b) não permeabilizadas. Estas células foram incubadas com PCs $0,10 \mathrm{mg} \mathrm{mL}^{-1}$ durante $12 \mathrm{~h}$ e lavados três vezes com PBS. Como controle negativo, algumas células foram incubadas com PBS. Essas análises foram realizadas usando o microscópio ZEISS Axio Scope.A1 com HBO 50, e as imagens foram capturadas usando o software ZEISS AxioCam MRc e AxioVision LE 4.8.1 no DAPI (BP 420-480 nm), FITC (BP 505-530 nm), e filtros de emissão de rodamina (Meta 560-615 nm). Também foi realizado o teste de in vitro de toxicidade, utilizando PCs nas concentrações de 0 (controle); 0,05; 0,10 e 0,20 mg mL-1. As células HEK293 foram incubadas por 6, 12, 24 e $48 \mathrm{~h}$ e em seguida, através do teste de exclusão com o corante azul de Tripan, foi obtido o percentual de células vivas e mortas, e esses dados foram testados através de ANOVA. Todos os ensaios biológicos foram realizados conforme descrito por Rocha et al. (2017).

As amostras de PCs foram caracterizadas por diferentes técnicas a fim de se obter informações sobre composição, estrutura e propriedades. A termogravimetria foi realizada em um equipamento DTG-60H da SHIMADZU e as amostras foram submetidas a um tratamento térmico de até $1000^{\circ} \mathrm{C}$ em atmosfera inerte. As informações sobre os grupos funcionais foram identificadas a partir da espectroscopia infravermelho com transformada de Fourier (IVTF). As análises foram realizadas a partir do espectrômetro Spectrum GX da Perkin-Elmer. Foram registrados espectros na região de 4000 a 400 $\mathrm{cm}^{-1}$, usando resolução de $4 \mathrm{~cm}^{-1}$ e acúmulo de 32 espectros. As amostras foram dispersas em $\mathrm{KBr}$ e pastilhadas com auxílio de uma prensa hidráulica usando-se 10 toneladas de pressão de compactação. Os espectros de absorção foram obtidos a partir de um espectrofotômetro com arranjo de diodos UV- 2550 SHIMADZU. As medidas foram realizadas em amostras na temperatura ambiente na região de 200 a $900 \mathrm{~nm}$, utilizando-se água como solvente e células de quartzo de 1 cm de caminho óptico. Os Espectros de Fotoluminescência foram monitorados com o espectrofluorímetro RF- 5301PC SHIMADZU. As medidas de emissão foram realizadas na região entre 350 e $750 \mathrm{~nm}$, com variação do comprimento de onda de excitação entre 330 a $420 \mathrm{~nm}$. As medidas foram conduzidas em células de quartzo de $1 \mathrm{~cm}$ de caminho óptico. Uma lâmpada de xenônio de $150 \mathrm{~W}$ foi empregada como fonte de excitação, sendo utilizadas fendas de $3 \mathrm{~nm}$ para excitação e emissão. As medidas do tempo de vida do estado excitado foram obtidas utilizando um espectrofluorímetro (Fluorolog-3, Horiba Jbin Yvon), equipado com um nanoled como fonte de excitação pulsada com comprimento de onda de $340 \mathrm{~nm}$, e duração de pico menor que $1.0 \mathrm{~ns}$. A taxa de repetição foi de $1.00 \mathrm{MHz}$ e a faixa de medida de $100 \mathrm{~ns}$, com 3850 canais com resolução de 2,74 x 10-2 ns/Chanel.

Para determinação do rendimento quântico de fotoluminescência foi utilizado um método comparativo entre uma solução padrão de um corante com $\Phi$ f conhecido e uma suspensão de PCs. Ambas as soluções foram preparadas e excitadas em $355 \mathrm{~nm}(\lambda \mathrm{exc})$. Foram realizados espectros de absorção das amostras, anotando o valor da intensidade de absorção no comprimento de onda de excitação. Concomitantemente, foram registrados espectros de emissão das amostras, coletando os valores da área integrada do espectro de fotoluminescência. Com os valores registrados, um gráfico de área integrada de fotoluminescência em relação à intensidade de fluorescência foi obtido. O módulo do coeficiente angular da reta obtida em cada gráfico foi utilizado para o cálculo dos valores de rendimentos quânticos de acordo com a Equação 1.

$$
\phi_{\mathrm{f}}=\phi_{\mathrm{ST}}\left(\mathrm{m}_{\mathrm{x}} / \mathrm{m}_{\mathrm{ST}}\right)\left(\left(\eta_{\mathrm{x}}^{2}\right) /\left(\eta_{\mathrm{ST}}^{2}\right)\right)
$$


Os subscritos x e ST são referentes a amostra teste, no caso os PCs, e a solução padrão, no caso o sulfato de quinino. O termo $\mathrm{m}$ é o coeficiente angular obtido pelo gráfico descrito anteriormente e $\eta$ é o índice de refração do solvente da amostra e da solução padrão. O sulfato de quinino $\left(\phi_{\mathrm{f}}=54 \%\right)$ foi escolhido pois, a substância padrão deve apresentar emissão e absorção em uma região similar da amostra a ser estudada.

\section{RESULTADOS E DISCUSSÃO}

A análise termogravimétrica do precursor ovalbumina foi realizada com o intuito de verificar a estabilidade térmica do mesmo e assim selecionar as faixas de temperaturas ideais para as sínteses dos PCs. A Figura 1 apresenta o termograma da decomposição térmica do precursor ovalbumina. É possível observar um evento de perda de massa que se estende da temperatura ambiente até $\sim 120^{\circ} \mathrm{C}$, atribuído à perda de água fisicamente absorvida [18]. Estudos sugerem que eventos entre 200 e $600^{\circ} \mathrm{C}$ caracterizam o intervalo da degradação do precursor, com perda de massa que está diretamente relacionada com a quebra das cadeias proteicas e na ruptura das ligações peptídicas [19, 20]. As temperaturas selecionadas para a síntese via pirólise e suas respectivas massas (\%) estão representadas na Tabela 1 , ressaltando que à medida que a temperatura aumenta uma grande quantidade em massa do material é perdida.

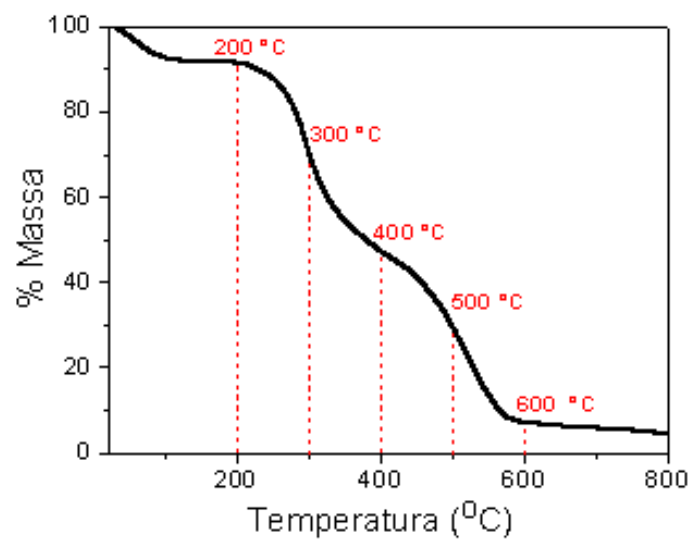

Figura 1: Curva termogravimétrica do precursor ovalbumina, até $1000^{\circ} \mathrm{C}\left(10^{\circ} \mathrm{C} / \mathrm{min}\right)$ em atmosfera inerte.

Tabela 1: Valores de temperatura selecionados para síntese e suas respectivas massas resultantes do tratamento térmico.

\begin{tabular}{cc}
\hline TEMPERATURA $\left({ }^{\circ} \mathbf{C}\right)$ & MASSA (\%) \\
\hline 200 & 90,94 \\
300 & 69,25 \\
400 & 40,50 \\
500 & 29,69 \\
600 & 6,96 \\
\hline
\end{tabular}

Além disso, com o intuito de obter informações sobre os grupos funcionais presentes nos PCs, foram registrados espectros infravermelho das amostras preparadas. Ao se observar os espectros infravermelho dos PCs apresentados na Figura 2, foi possível averiguar inicialmente que cada método de síntese gerou amostras com comportamentos diferentes, o que pode ser devidamente comprovado ao se comparar os perfis espectrais, assim como o deslocamento das bandas dos grupos funcionais, em ambas as rotas de síntese. 
a)

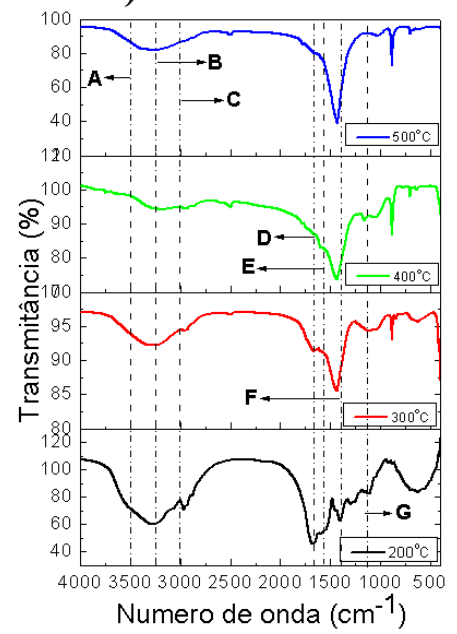

b)

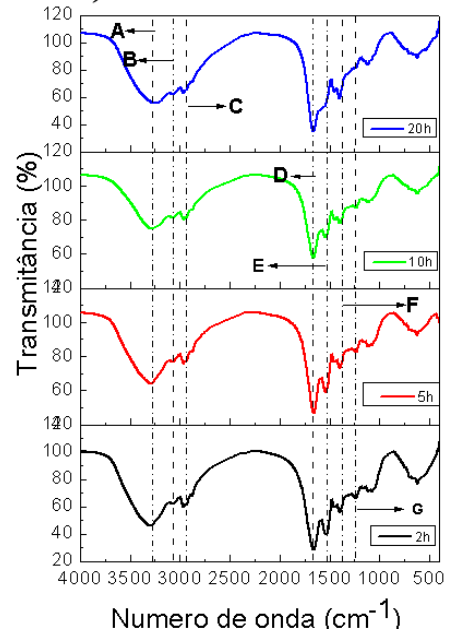

Figura 2: Espectros IVTF das amostras de PCs: a) via pirólise b) via rota hidrotérmica. As letras de identificação das bandas estão identificadas na Tabela 2 e 3 , respectivamente.

Foram identificadas absorções características na região de $3500-3100 \mathrm{~cm}^{-1}$ de banda larga atribuída ao estiramento $\mathrm{O}-\mathrm{H}$, indicando a presença de grupos hidroxilas; estiramento assimétrico e dobramento assimétrico das ligações N-H em $\sim 3233$ e $1560 \mathrm{~cm}^{-1}$, respectivamente, indicando a presença de grupos funcionais amina. É possível observar absorções normalmente atribuídas as ligações $\mathrm{C}-\mathrm{H}$ de grupos $\mathrm{CH}_{3} \sim 2965 \mathrm{~cm}^{-1}$, vibrações de estiramento $\mathrm{C}=\mathrm{O} \mathrm{em} \sim 1677 \mathrm{~cm}^{-1}$, indicando a presença de ácido carboxílico além de outros grupos contendo oxigênio e os estiramentos $\mathrm{CH}_{2}$ de alcanos $\sim 1450$ $\mathrm{cm}^{-1}$ e C-C em $1142 \mathrm{~cm}^{-1}$. Para os PCs obtidos via pirólise (Figura 2a), foi possível observar que os estiramentos O-H e $\mathrm{C}=\mathrm{O}$ estão bem definidos no espectro de $200{ }^{\circ} \mathrm{C}[21,22]$, porém, à medida que a temperatura aumenta, em específico a 400 e $500{ }^{\circ} \mathrm{C}$, estes grupos funcionais se degradam, não podendo ser mais visualizados nos espectros. As frequências e atribuições estão apresentadas na Tabela 2.

Tabela 2: Principais vibrações características dos grupos moleculares de PCs obtidos a partir da ovalbumina via pirólise.

\begin{tabular}{ccc}
\hline IDENTIFICAÇÃO & POSIÇÃO DAS BANDAS $\left(\mathbf{C M}^{-1}\right)$ & ATRIBUIÇÃO \\
\hline A & 3500 & $v$ O-H \\
B & $\sim 3233$ & $v_{\text {ass }} \mathrm{N}-\mathrm{H}$ \\
C & $\sim 2965$ & $v_{\text {ass }} \mathrm{C}-\mathrm{H}$ \\
D & $\sim 1677$ & $v \mathrm{C}=\mathrm{O}$ \\
E & $\sim 1560$ & $\delta_{\text {ass }} \mathrm{N}-\mathrm{H}$ \\
F & $\sim 1450$ & $v \mathrm{CH}_{2}$ \\
G & $\sim 1142$ & $v$ C-C \\
\hline
\end{tabular}

$v$ : estiramento; $v_{\text {ass }}$ : estiramento assimétrico; $\delta_{\text {ass }}$ : dobramento assimétrico

No caso do método hidrotérmico, não foi possível observar quaisquer mudanças significativa nos perfis dos espectros, quando comparados entre si. Acredita-se que os grupos presentes nos PCs estão localizados em sua superfície com exceção da vibração $\mathrm{C}=\mathrm{C}$ aromática que além de estar na superfície é característica do núcleo dos PCs [23]. A Tabela 3 apresenta as vibrações presentes nas amostras sintetizadas por esse método. 
Tabela 3: Principais vibrações características dos grupos moleculares de PCs obtidos a partir da ovalbumina via rota hidrotérmica.

\begin{tabular}{ccc}
\hline IDENTIFICAÇÃO & POSIÇÃO DAS BANDAS $\left(\mathbf{C M}^{-1}\right)$ & ATRIBUIÇÃO \\
\hline A & $\sim 3280$ & $v$ O-H \\
B & $\sim 3074$ & $v$ ass N-H \\
C & $\sim 2937$ & $v$ C-H \\
D & $\sim 1653$ & $v$ C $=\mathrm{O}$ \\
E & $\sim 1530$ & $\delta_{\text {ass }}$ N-H \\
F & $\sim 1377$ & $v$ C sp \\
G & $\sim 1240$ & $v$ C-C \\
\hline
\end{tabular}

$v$ : estiramento; $v_{\text {ass }}:$ estiramento assimétrico; $\delta_{\text {ass }}:$ dobramento assimétrico

Como visto nos espectros de infravermelho, os PCs são compostos majoritariamente por elementos como carbono, hidrogênio, nitrogênio e oxigênio. Dessa forma, as transições eletrônicas das nanopartículas estão diretamente ligadas as de moléculas orgânicas. As transições mais comuns para materiais orgânicos são: $\pi-\pi^{*}, n-\pi^{*}, \sigma-\sigma^{*}, \sigma-\pi^{*}$ e $n-\sigma^{*}$. As transições do tipo $\sigma-\sigma^{*}$ e n- $\sigma^{*}$ são raramente observadas em espectros de materiais de carbono, devido à alta energia necessária para a ocorrência da transição. Já as transições $\pi-\pi^{*}$ e $n-\pi^{*}$, são as mais encontradas, pois, as energias de transições nesses casos são mais baixas e, portanto, mais facilmente acessíveis [21]. A Figura 3 apresenta os espectros UV-Vis dos PCs preparados via pirólise em diferentes temperaturas.

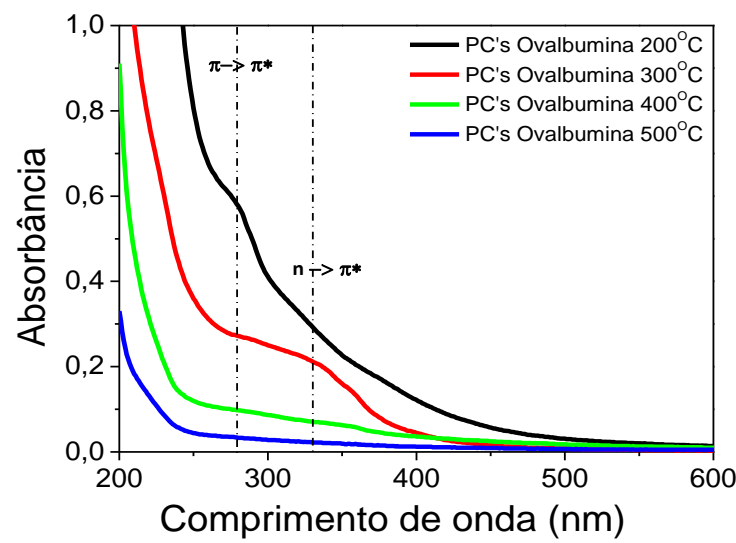

Figura 3: Espectros de absorção das amostras de PCs derivados da ovalbumina obtidos em diferentes temperaturas via pirólise.

De acordo com os espectros apresentados na Figura 3, nota-se que as nanopartículas sintetizadas a $200^{\circ} \mathrm{C}$ e $300{ }^{\circ} \mathrm{C}$ possuem uma banda larga devido à transição n- $\pi^{*}$ e outra devido à transição $\pi-\pi^{*}$ (entre 250 e $300 \mathrm{~nm}$ ). As transições de $\mathrm{n}-\pi^{*}$ são características de grupos de superfície do tipo $\mathrm{C}-\mathrm{O}$ e $\mathrm{C}=\mathrm{O}$, os quais foram observados nos espectros de infravermelho. Já as transições $\pi$ - $\pi^{*}$ correspondem a duplas ligações do átomo de carbono [21]. Analisando o comportamento da absorção, percebe-se que com o aumento da temperatura de pirólise a intensidade de absorção diminui, pois, o material se aproxima de uma carbonização completa. Isso é confirmado pelos materiais preparados a $400^{\circ} \mathrm{C}$ e $500{ }^{\circ} \mathrm{C}$, os $\mathrm{PCs}$ apresentam apenas uma leve banda de absorção $\pi-\pi^{*}$. A não identificação da transição $n-\pi^{*}$ para estas temperaturas também pode ser explicado pelo aumento da temperatura de síntese, provocando a degradação dos grupos funcionais que se encontram presentes na superfície dos PCs [24]. Já nos espectros das amostras preparadas via hidrotérmica, apresentados na Figura 4, é possível perceber uma banda de absorção na região entre 250 e $300 \mathrm{~nm}$ e uma leve inflexão na região mais à frente. Esta banda, que se torna mais intensa a cada variação do tempo de síntese, indica a transição $\pi-\pi^{*}$, o que corresponde, portanto, a transições de duplas ligações [21]. O aumento da intensidade de absorção com o aumento do tempo de síntese, pode estar relacionado com a melhor formação das nanopartículas, ou seja, para esse precursor utilizado, quanto maior o tempo de síntese do material a $150^{\circ} \mathrm{C}$, melhor para a formação dos PCs. 


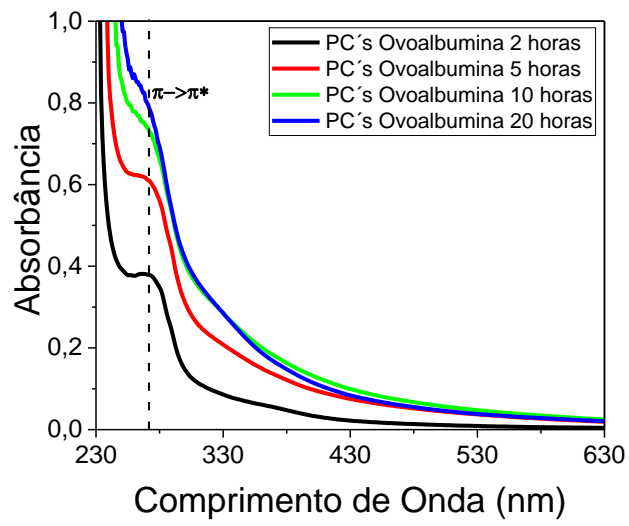

Figura 4: Espectros de absorção das amostras de PCs derivados da ovalbumina obtidos em diferentes tempos de síntese hidrotérmica a $150^{\circ} \mathrm{C}$.

A espectroscopia de fotoluminescência foi empregada para a caracterização óptica dos PCs, a fim de se obter informações sobre os processos de emissão das nanopartículas de carbono. A Figura 5 apresenta os espectros de fotoluminescência dos PCs sintetizados via pirólise. Os espectros apresentaram emissão típica dos PCs na região do azul e variações de acordo com comprimento de onda de excitação, isso se deve ao fato da presença de diversos grupos de superfície, o que é característica do precursor utilizado.
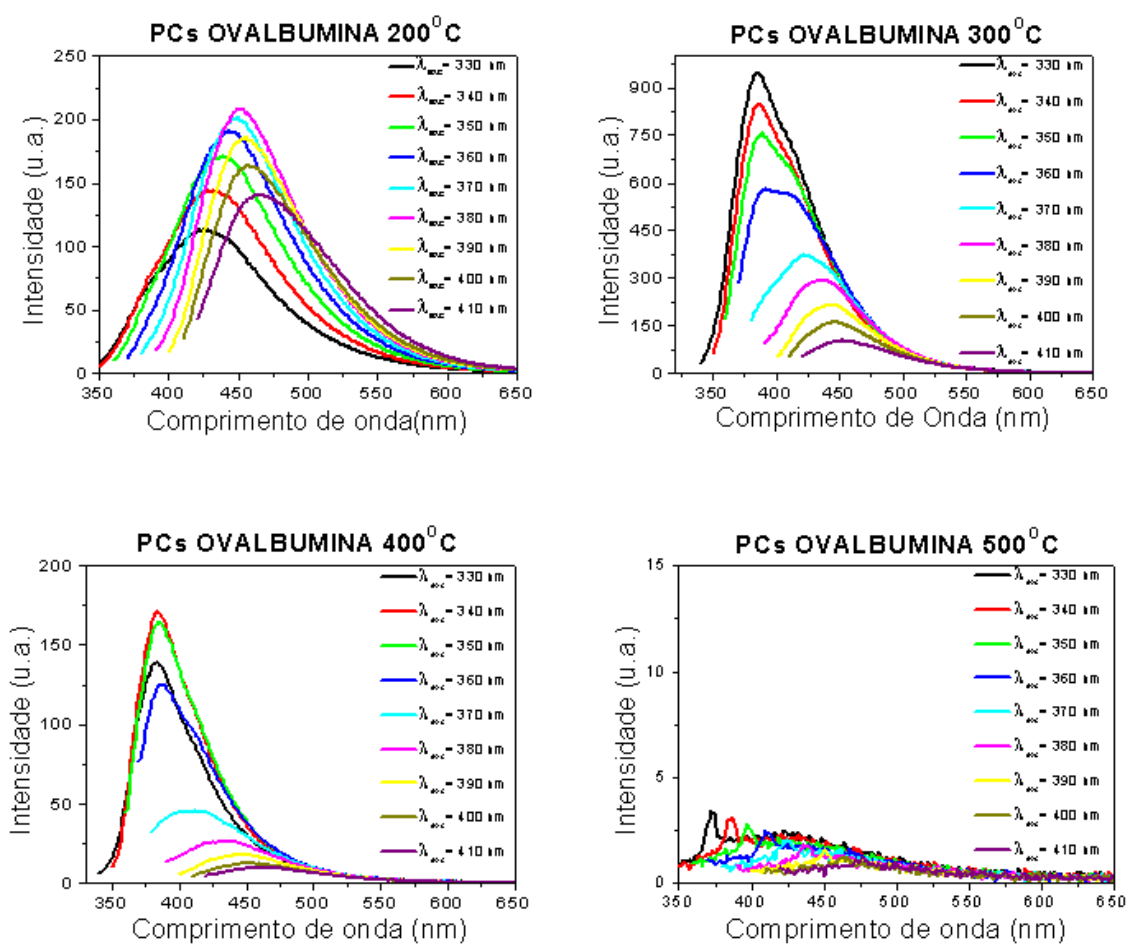

Figura 5: Espectros de fotoluminescência das amostras de PCs obtidos em diferentes temperaturas via pirólise, em função dos diferentes comprimentos de onda de excitação.

Para a amostra de $500{ }^{\circ} \mathrm{C}$, nenhuma emissão foi observada. Isso pode ser explicado por uma possível degradação dos grupos funcionais presentes no precursor, devido a temperatura considerada extrema para este tipo de material, ou seja, essa temperatura não é favorável para a formação das nanopartículas. Nas amostras sintetizadas via hidrotérmica, o comportamento dos espectros é semelhante aos da pirólise, apresentando um deslocamento para a região do vermelho com a mudança do comprimento de onda de excitação. A Figura 6 apresenta os espectros de fotoluminescência dessas amostras. 

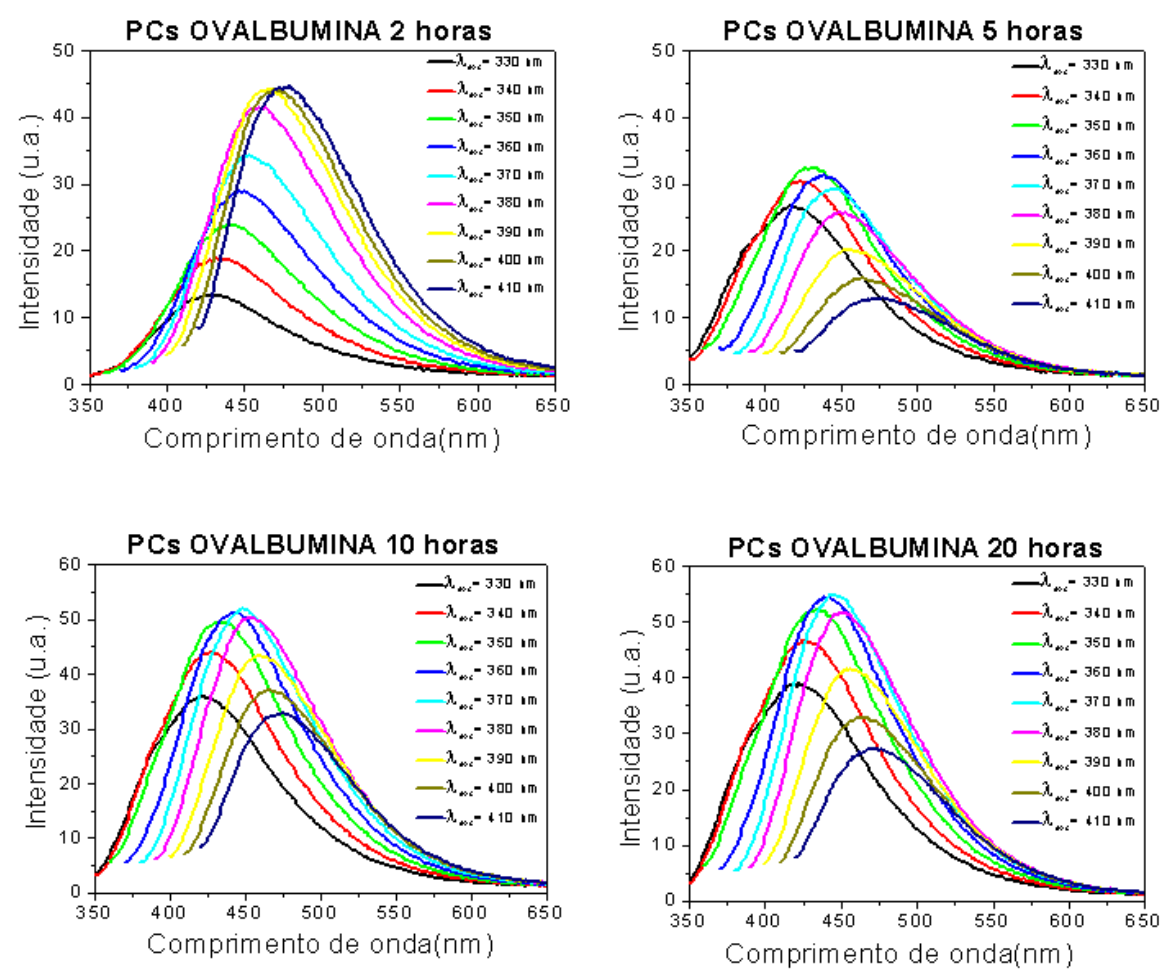

Figura 6: Espectros de fotoluminescência das amostras de PCs obtidos em diferentes tempos via hidrotérmica $\left(150^{\circ} \mathrm{C}\right)$, em função dos diferentes comprimentos de onda de excitação.

Para saber a probabilidade do estado excitado ser desativado pelo fenômeno da fluorescência, foram realizados os cálculos do rendimento quântico $\left(\Phi_{\mathrm{f}}\right)$ das amostras. Esse cálculo é a razão do número de fótons emitidos pelo número de fótons absorvidos. Além disso foram realizadas medidas de tempo de decaimento do estado excitado, com o intuito de avaliar o mecanismo do processo de decaimento radiativo dos nanomateriais. As Tabelas 4 e 5 apresentam os valores de $\Phi_{\mathrm{f}}$ e tempo de vida para os PCs obtidos via pirólise e via hidrotérmica, respectivamente. É possível observar que os PCs via pirólise, sintetizados na temperatura mais elevada não obtiveram valor de $\Phi_{\mathrm{f}}$ devido a sua baixa ou quase inexistente fluorescência, o que é comprovado pelo tempo de vida não ser possível de calcular. Essas informações corroboram com a teoria de que temperaturas mais brandas, nesse método de síntese, favorece uma melhor formação dos PCs, ocorrendo neste caso uma carbonização incompleta.

Tabela 4: Valores de tempo de vida e $\Phi_{\mathrm{f}}$ dos PCs obtidos via pirólise.

\begin{tabular}{ccc}
\hline AMOSTRA & TEMPO DE VIDA (ns) & $\boldsymbol{\Phi}_{\mathbf{f}}(\%)$ \\
\hline PC $200^{\circ} \mathrm{C}$ & 3,39 & 9,0 \\
PC $300^{\circ} \mathrm{C}$ & 3,23 & 6,7 \\
PC $400^{\circ} \mathrm{C}$ & 3,38 & 6,3 \\
PC $500^{\circ} \mathrm{C}$ & - & - \\
\hline
\end{tabular}

Nas amostras sintetizadas via hidrotérmica, os PCs preparados em menores tempos de síntese apresentaram menores valores de $\Phi_{\mathrm{f}, \text {. }}$ Como o $\Phi_{\mathrm{f}}$ é uma relação de fótons emitidos por fótons absorvidos, as amostras que apresentaram maiores intensidades de absorção, apresentaram os menores valores de $\Phi_{\mathrm{f}}$ uma vez que neste caso todos os PCs exibiram emissões semelhantes.

Tabela 5: Valores de tempo de vida e $\Phi_{\mathrm{f}}$ dos PCs obtidos via hidrotérmica a $150^{\circ} \mathrm{C}$.

\begin{tabular}{ccc}
\hline AMOSTRA & TEMPO DE VIDA (ns) & $\boldsymbol{\Phi}_{\mathbf{f}}(\%)$ \\
\hline PC 2h & 3,39 & 5,4 \\
PC 5h & 3,78 & 4,0 \\
PC 10 h & 3,34 & 3,9 \\
PC 20 h & 3,42 & 3,1 \\
\hline
\end{tabular}


Analisando as informações obtidas, as duas amostras com maiores valores de $\Phi_{\mathrm{f}}$ (uma de cada método de síntese) foram escolhidas para serem utilizadas como marcadores celulares (pirólise PC $200^{\circ} \mathrm{C}$ e hidrotérmica PC $2 \mathrm{~h}$ ). A Figura 7 mostra que os PCs obtidos via hidrotérmica foram capazes de interagir com as células HEK293 permeabilizadas e não permeabilizadas e apresentam intensa fluorescência no verde (filtro FITC), não sendo observada emissão no vermelho. Essa boa interação se deve a natureza dos PCs, aos seus muitos grupos funcionais ativos, além de também demonstrar que esse material é capaz de atravessar a membrana plasmática das células HEK293, indicando o potencial promissor desses materiais na área biológica. No entanto, não houve a mesma interação das células entre essas células e os PCs obtidos via pirólise (Figura 8).

a)

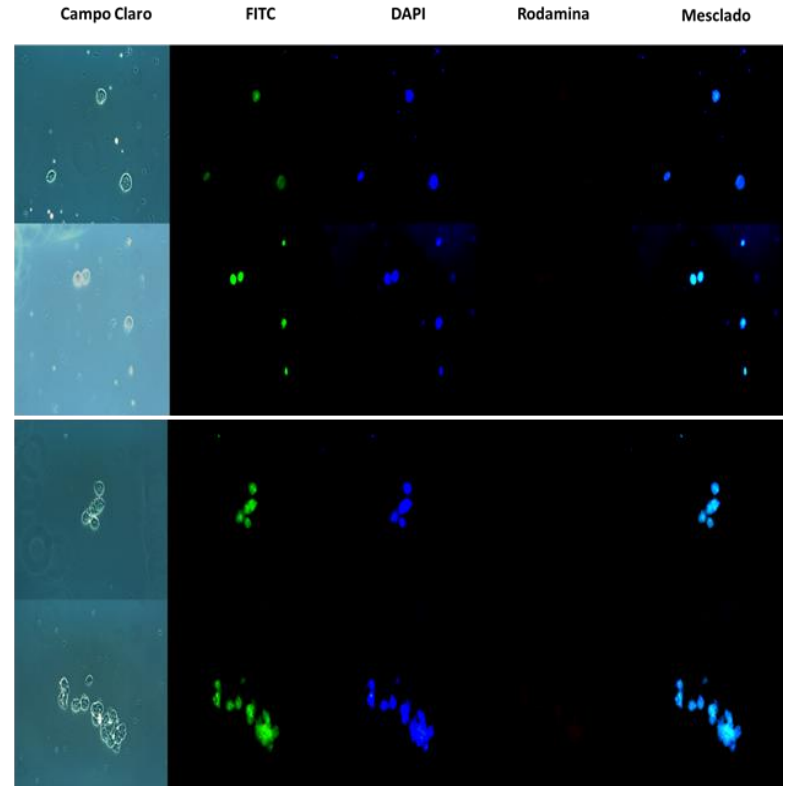

Figura 7: Imagens de fluorescência de células HEK293 contendo $0,1 \mathrm{mg} \mathrm{mL}^{-1}$ de $\mathrm{PCs}$ obtidos via hidrotérmica a $150^{\circ} \mathrm{C}$ por $2 \mathrm{~h}$. a) sem permeabilização b) permeabilizadas.

Apesar de um maior valor de $\Phi_{\mathrm{f}}$, valor usado como parâmetro para a utilização como sondas de outros materiais [16], os PCs não conseguiram a interação satisfatória para a obtenção de boas imagens e isso pode estar relacionado com o método de síntese utilizado e à presença de grupos funcionais na superfície. As amostras preparadas pela rota de pirólise são submetidas à temperatura de $200^{\circ} \mathrm{C}$ por $2 \mathrm{~h}$, e, assim essa etapa pode decompor alguns grupos funcionais, os quais poderiam interagir melhor com as células HEK293 estudadas, neste caso. Ao se comparar os espectros de IVTF, o método hidrotérmico apresenta mais bandas vibracionais, se assemelhando mais àqueles de moléculas orgânicas. No método hidrotérmico, a temperatura utilizada na síntese é $150^{\circ} \mathrm{C}$ e tempo de patamar, que para essa amostra foi de $2 \mathrm{~h}$, a amostra passa por um processo mais simples de purificação que aquela preparada pelo método de pirólise. Essa etapa de purificação e a utilização de uma temperatura mais baixa, também pode interferir na melhor interação das nanopartículas com as células.

a)

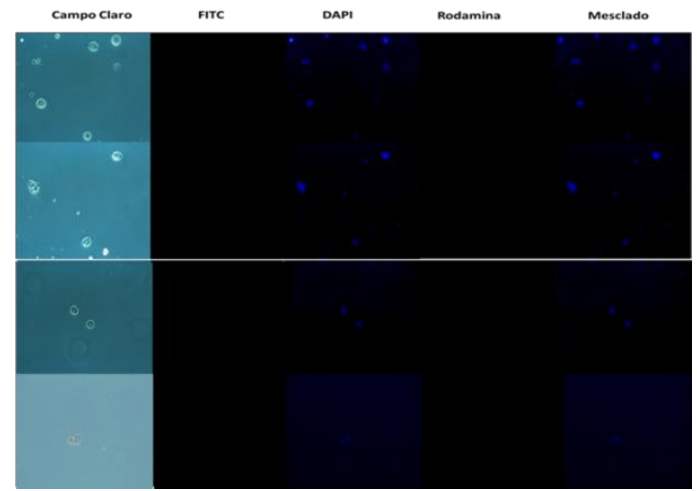

Figura 8: Imagens de fluorescência de células HEK293 contendo $0,1 \mathrm{mg} \mathrm{mL}^{-1}$ de PCs obtidos via pirólise a $200^{\circ} \mathrm{C}$. a) sem permeabilização b) permeabilizadas. 
Para avaliar a biocompatibilidade o teste de viabilidade foi realizado. Como demonstrado na Figura 9, o índice de viabilidade das células não apresenta alterações significativas até concentrações de $0,20 \mathrm{mg} \mathrm{mL}^{-1}$. Ao se analisar os resultados para as duas amostras, percebe-se que ambas as nanopartículas sintetizadas apresentam valores de viabilidade em torno de $60 \%$ seja ao longo do tempo de análise ou do aumento de concentração. Estes resultados podem ser atribuídos à baixa citotoxicidade dos PCs promovendo uma interação que não induz a morte celular, confirmando que o material é promissor para a utilização na marcação celular.

a)

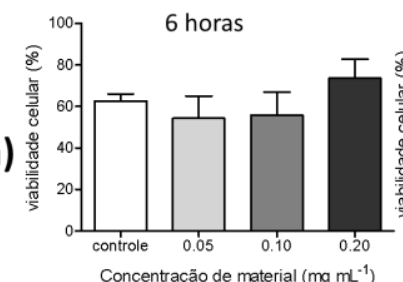

6 horas

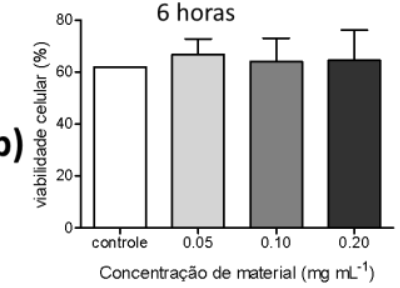

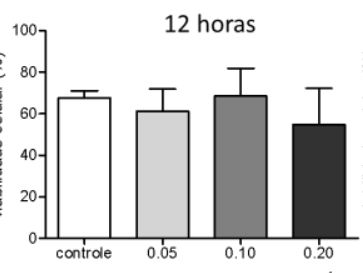

Concentração de material $\left(\mathrm{mg} \mathrm{mL}^{-1}\right)$

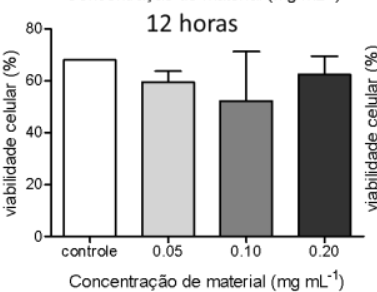

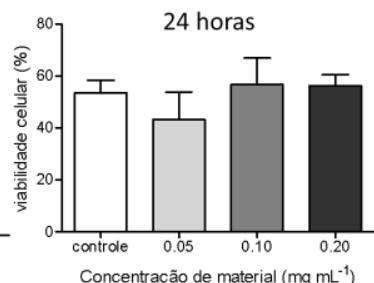

24 horas

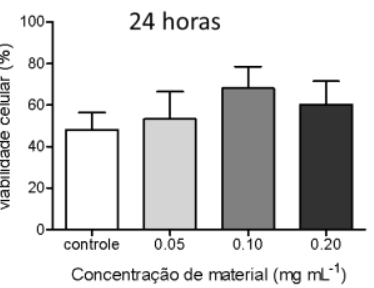

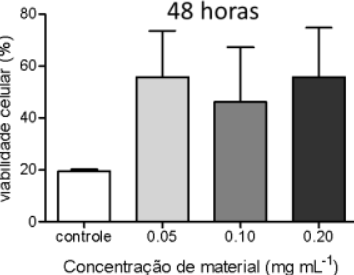

48 horas

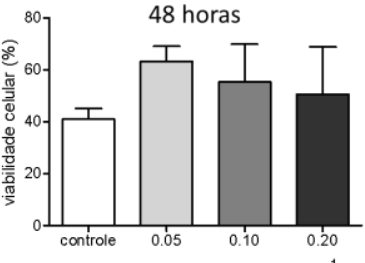

Figura 9: Índice de viabilidade das células HEK293 incubadas com a) PCs obtidos via pirólise b) PCs obtidos via hidrotérmica.

\section{CONCLUSÕES}

Os métodos de síntese utilizados foram adequados para a produção dos materiais, que apresentaram luminescência na região do azul e emissão dependente do comprimento de onda de excitação. Para os PCs obtidos via pirólise e via hidrotérmica a $150^{\circ} \mathrm{C}$, observou-se que temperaturas mais altas não favorecem a formação das nanopartículas pela rota de pirólise e, maiores tempos de síntese, no caso da via hidrotérmica, tornaram o material um bom absorvedor, porém com menor emissão. Apesar de apresentar o menor valor de rendimento quântico, a amostra obtida via hidrotérmica exibiu melhor interação com células estudadas, apresentando luminescência intensa na região do verde. Essa melhor interação mostra que os grupos de superfície podem influenciar na interação com as células, já que essas amostras apresentaram espectros de infravermelho com perfis diferentes dos PCs sintetizados via pirólise. O mecanismo de imagem entre os PCs e células animais ainda estão em debate e estudos precisam ser aprofundados para melhor entendimento. Neste trabalho foi possível demostrar que amostras de PCs preparadas via rota hidrotérmica apresentaram melhor perfil de interação com células HEK293. Além disso, foi possível observar que as nanopartículas de carbono preparadas são promissoras na utilização em bioimagem e não induziram a morte das células, mostrando assim potencial para as aplicações apresentadas neste trabalho.

\section{AGRADECIMENTOS}

A CNPq, CAPES, FINEP e FAPEMIG pelo apoio e financiamento ao trabalho.

\section{BIBLIOGRAFIA}

[1] FARSHBAF, M., et al. "Carbon quantum dots: recent progresses on synthesis, surface modification and applications”. Artificial Cells, Nanomedicine, And Biotechnology, v. 46, n. 7, p.1331-1348, Sep. 2017.

[2] ZHU, Z., et al. "Red carbon dots: Optical property regulations and applications". Materials Today, v. 30, p.52-79, Nov. 2019.

[3] ZARBIN, A.J.G.; OLIVEIRA, M.M. "Nanoestruturas de carbono (nanotubos, grafeno): Quo Vadis?" Química Nova, v. 36, n. 10, p.1533-1539, Aug. 2013.

[4] MOLAEI, M.J. “A review on nanostructured carbon quantum dots and their applications in biotechnology, sensors, and chemiluminescence”. Talanta, v. 196, p.456-478, May. 2019.

[5] SUN, X., LEI, Y. "Fluorescent carbon dots and their sensing applications". Trac Trends In Analytical Chemistry, v. 89, p.163-180, Apr. 2017. 
[6] MEILING, T.T., et al. "Photophysics and Chemistry of Nitrogen-Doped Carbon Nanodots with High Photoluminescence Quantum Yield.” The Journal Of Physical Chemistry C, v. 122, n. 18, p.10217-10230, Apr. 2018.

[7] CHEN, B.B., et al. "Fluorescent carbon dots functionalization". Advances In Colloid And Interface Science, v. 270, p.165-190, Aug. 2019.

[8] YAO, B., et al. “Carbon Dots: A Small Conundrum”. Trends In Chemistry, v. 1, n. 2, p.235-246, May. 2019.

[9] LI, H., et al. "Carbon nanodots: synthesis, properties and applications". Journal Of Materials Chemistry, v. 22, n. 46, p.24230-24253, Dec. 2012.

[10] GHOSAL, K., GHOSH, A. "Carbon dots: The next generation platform for biomedical applications". Materials Science And Engineering: C, v. 96, p.887-903, Mar. 2019.

[11] AMDARI, P., et al. "Synthesis, properties and biomedical applications of carbon-based quantum dots: An updated review". Biomedicine \& Pharmacotherapy, v. 87, p.209-222, Mar. 2017.

[12] DENG, Q., et al. "Ruthenium nitrosyl grafted carbon dots as a fluorescence-trackable nanoplatform for visible light-controlled nitric oxide release and targeted intracellular delivery". Journal Of Inorganic Biochemistry, v. 165, p.152-158, Dec. 2016.

[13] HU, X., et al. "Easy synthesis of highly fluorescent carbon dots from albumin and their photoluminescent mechanism and biological imaging applications”. Materials Science And Engineering: C, v. 58, p.730736, Jan. 2016.

[14] LI, K., et al. "Technical synthesis and biomedical applications of graphene quantum dots". Journal of Materials Chemistry B, v. 5, p. 4811-4826, Oct.2017.

[15] DU, J., et al. "Carbon Dots for In Vivo Bioimaging and Theranostics”, Small, Feb. 2019, 1805087.

[16] JHONSI, M.A. "Carbon Quantum Dots for Bioimaging”. State Of The Art In Nano-bioimaging, p.35-53, Jun. 2018.

[17] MARTÍNEZ-MONGE, I., et al., "A new strategy for fed-batch process control of HEK293 cell cultures based on alkali buffer addition monitoring: comparison with O.U.R. dynamic method". Applied Microbiology And Biotechnology, v. 102, n. 24, p.10469-10483, Oct. 2018.

[18] YUSLIZA, Y., ZURAIDA, A., "The Effect of Fibres Content on Cotton Reinforced Albumen Composites”. Engineering Journal, Vol.12, No.2, Feb. 2011.

[19] SOMANATHAN, N., et al., "Thermal stability of modified caseins". Thermochim Acta, v. 302, p.47-52, Out. 1997.

[20] PUREVSUREN, B., DAVAAJAV, Y., “Thermal analysis of casein”. J Therm Anal Calorim, vol. 65, p. 147-152. Sep. 2001.

[21] PAVIA, D.L., LAMPMAN, G.M., KRIZ, G.S., et al., Introdução à Espectroscopia, 4a ed. São Paulo: Cengage Learning, 2010.

[22] SILVERSTEIN, R.M., WEBSTER, F.X., KIEMLE, D.J., Spectrometric Identification of Organic Compounds, $7^{\mathrm{a}} \mathrm{Ed}$, Wiley, 2002.

[23] Liang, Q., et al. "Easy synthesis of highly fluorescent carbon quantum dots from gelatin and their luminescent properties and applications”. Carbon, v.60, 421-428, Mar. 2013.

[24] YAO, B., et al. "Carbon Dots: A Small Conundrum”. Trends In Chemistry, v. 1, n. 2, p.235-246, May 2019.

ORCID

Sarah Lima

Luis Fernando T. Domingos

Roberto Vaz

Paulo Henrique de Almeida Campos Junior

Marco Antônio Schiavon https://orcid.org/0000-0002-0567-4843

https://orcid.org/0000-0003-2605-1220

https://orcid.org/0000-0003-0459-8153

https://orcid.org/0000-0003-2400-2548

https://orcid.org/0000-0002-1553-5388 